\title{
Myocardial contrast echocardiography assessment of mouse myocardial infarction: Comparison of kinetic parameters with conventional methods
}

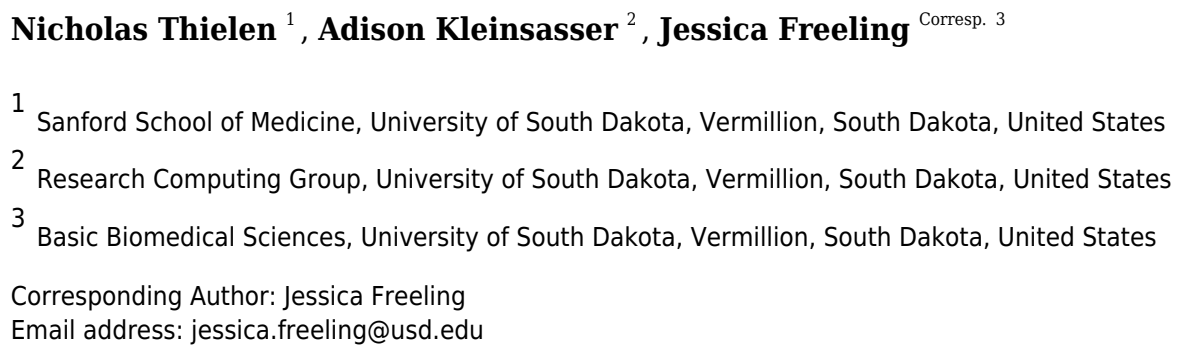

This study explores the use of a minimally invasive assessment of myocardial infarction (MI) in mice using myocardial contrast echocardiography (MCE). The technique uses existing equipment and software readily available to the average researcher. C57/BL6 mice were randomized to either MI or sham surgery and evaluated using MCE at 1- or 2weeks post-surgery. Size-isolated microbubbles were injected via retro-orbital catheter where their non-linear characteristics were utilized to produce the two-dimensional parameters of Wash-in-Rate and the Peak Enhancement, indicative of relative myocardial perfusion and blood volume, respectively. Three-dimensional cardiac reconstructions allowed the calculation of the Percent Agent, interpreted as the vascularity of the entire myocardium. These MCE parameters were compared to conventional assessments including M-Mode, strain analysis, and 2,3,5-Triphenyltetrazolium chloride (TTC) staining. Except for the Wash-in-Rate 2-week cohort, all MCE parameters were able to differentiate sham-operated versus $\mathrm{MI}$ animals and correlated with TTC staining $(P<0.05)$. MCE parameters were also able to identify $\mathrm{Ml}$ group animals which failed to develop infarctions as determined by TTC staining. This study provides basic validation of these MCE parameters to detect $\mathrm{MI}$ in mice complementary to conventional methods while providing additional hemodynamic information in vivo. 
1

2

3

4

5

6

7

8

9

10

11

12

13

14

15

16

17

18

19

20

21

22

23
Title

Myocardial Contrast Echocardiography Assessment of Mouse Myocardial Infarction: Comparison of Kinetic Parameters with Conventional Methods

\author{
Authors \\ Nicholas Thielen ${ }^{1}$, Adison Kleinsasser ${ }^{2}$, Jessica L. Freeling ${ }^{3}$ \\ ${ }^{1}$ University of South Dakota, Sanford School of Medicine, Vermillion, SD \\ ${ }^{2}$ University of South Dakota, Research Computing Group, Vermillion, SD \\ ${ }^{3}$ University of South Dakota, Basic Biomedical Sciences, Vermillion, SD
}

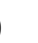

\section{Corresponding Author}

Jessica Freeling

414 E. Clark Street

Vermillion, SD 57069

jessica.freeling@usd.edu 
24

25

26

27

28

29

30

\section{Introduction}

Cardiovascular diseases are the leading cause of death globally, affecting an estimated 17.9 million people in 2016 (WHO 2017). With such a high burden on society, there is an increasing demand for basic translational cardiovascular research to identify mechanisms and treatment options. Due to the high incidence of coronary artery disease, advances in pre-clinical research methods are important, particularly in relevant and widely applied animal models. The murine model is widely used (Michael et al. 1995) for human cardiovascular disease because of the physiological similarities, and rapidly expanding availability, of genetic modification tools (Scherrer-Crosbie \& Thibault 2008; Wang et al. 2006). This presents the need for physiologically relevant methods to accurately assess myocardial infarction (MI) in mice.

Currently validated methods of assessing MI with functional echocardiographic data offer a variety of limitations which could be improved upon with the addition of a perfusion imaging modality (Bhan et al. 2014) (Chen et al. 2016). Recent developments into the understanding of coronary microvascular disease further emphasize the need for accurate perfusion imaging as functional imaging may not detect coronary microvascular disease (Taqueti \& Di Carli 2018). Furthermore, coronary microvascular disease may play a role in stunned and hibernating myocardium and require the detection of preserved myocardial perfusion to make this diagnosis (Kloner 2020). The most commonly utilized strain for MI research, the C57BL/6 mouse, is known to exhibit multiple left anterior descending artery (LAD) phenotypic patterns, making consistency of MI pattern, and thus infarct area, particularly challenging (Ahn et al. 2004). Therefore, the development of a technique that can accurately assess parameters of vascularity of the myocardial wall would provide researchers with a more robust quantification of MI. This 
47 would enable the testing of longitudinal treatment paradigms and provide further insight into the

48 effects of both epicardial and micro-vascular disease on health outcomes.

49

50 Considering the availability and minimally invasive nature of ultrasound, myocardial contrast

51 echocardiography (MCE) may be a useful modality to assess myocardial perfusion. It is currently

52 a mobile and widely available imaging modality that can assess myocardial blood flow and

53 myocardial blood volume in vivo without ionizing radiation (Kaul 2008; Verkaik et al. 2018).

54 MCE has been established in humans and is typically performed alongside 2D B-Mode imaging

55 (Porter et al. 2020). Techniques in patients often utilize bolus injection or continuous infusions of

56 microbubbles that require a high mechanical index burst sequence and subsequent replenishment

57 analysis (Averkiou et al. 2020). Indeed, the use of MCE in dogs and rats to measure visual

58 perfusion defects have been validated (Chen et al. 2009; Dourado et al. 2003). These techniques

59 benefit from the relatively large size of the animal allowing enhanced visualization of

60 microbubbles in the myocardium but entail a high degree of subjectivity in assessing the

61 perfusion defect.

62

The use of MCE to evaluate mouse MI is well established. An early study validated MCE in

64 mice with a correlation of myocardial video intensity to Evans blue staining (Scherrer-Crosbie et al. 1999). More recent advances involve creating a 3D rendering with MCE which could visually be compared with postmortem histology staining (French et al. 2006). Another method of

67 quantifying MI with MCE has been performed by plotting the video intensity of a one-pixel-wide

68 line against time to indicate the perfusion of the myocardium (Alvarez et al. 2018). These

69 methods continue to validate the use of MCE in mice; however, they offer multiple areas that can 
be improved upon. Notably, none of them use an objective parameter to quantify the non-linear contrast signal given off from the myocardium.

The use of MCE in mice presents several issues, most of which involve the inherently small size of the heart. In mice, high-frequency ultrasound is required for imaging to facilitate sufficient resolution for accurate quantification, without excessive penetration. The higher frequencies required for mice therefore reduces both the ability to utilize harmonic imaging with microbubbles (Scherrer-Crosbie \& Thibault 2008), and the ability to penetrate through the opacified left ventricular (LV) chamber to visualize the posterior wall. These were reported to be resolved with methods that involved lodging large size isolated microbubbles within the myocardium after the lumen cleared (Kaufmann et al. 2007); however, this technique has not yet been reproduced. Most published techniques rely on subjective measurements of video/pixel intensity rather than measuring non-linear contrast (NLC) signal. Also, the protocols involved in these techniques and post-imaging analyses rely on complex self-made mathematical models or software programs that are not readily available, making them too time consuming and difficult to be performed in an efficient manner for the average cardiovascular researcher.

Here, we evaluate a comprehensive method that can identify and further characterize mouse MI using 2D and 3D NLC MCE. We utilized a commonly available ultrasound machine and postimaging software, employed the most widely used anesthesia regimen, and optimized the workflow, all of which will enable researchers to perform these techniques proficiently. Moreover, this technique detects in vivo MI in 3D by reporting the Percent Agent (PA), taking advantage of the non-linear nature of the microbubbles to report an objective value reflecting 
93 hemodynamic real-time information of the entire myocardium. Importantly, these MCE

94 parameters were validated and correlated both with 2,3,5-Triphenyltetrazolium chloride (TTC)

95 staining of ex vivo myocardium and in vivo conventional functional ultrasound measurements.

96 Thus, this minimally invasive technique uses size-isolated microbubbles (SIMB) and NLC MCE

97 to identify MI and provide relevant hemodynamic information about myocardial perfusion,

98 enabling enhanced characterization of the mouse MI model.

\section{Materials and Methods}

101

102

103

104

105

106

107

108

109

110

111

112

113

114

115

\section{Animal Model}

The University of South Dakota Institutional Animal Care and Use Committee approval was obtained for all experiments, IACUC \# 12-12-17-20D. We used a total of 28 female C57BL/6NHsd mice in this study (Envigo, Denver, CO) as this is the most widely utilized strain in cardiovascular research. All animals were housed in an American Association for Accreditation of Laboratory Animal Care accredited facility kept within appropriate temperature and humidity levels and exposed to 12/12 light/dark cycle. Mice were housed in microisolator cage racks, fed standard rodent chow ad libidum, and provided with environmental enrichment in the form of cotton bedding squares and plastic huts. Under general isoflurane anesthsia, 12 animals were randomly selected for sham surgery and 16 randomly selected for permanent LAD coronary artery ligation using an established surgical technique (Tarnavski et al. 2004). Buprenorphine SR LAB (Zoopharm, Laramie, WY) $1.0 \mathrm{mg} / \mathrm{kg}$ SQ given once, lasting 72 hours was given as analgesia prior to the animal regaining consciousness. Animals were monitored twice daily for signs of distress, pain, or dehydration in the three days after surgery. While the IACUC protocol included provision to euthanize animals if they exhibited any of these 
116 symptoms, no mice in this study met criteria for euthanasia. However, two mice died during

117 surgery due to hemmorhage and six mice died in the immediate post-surgical period of acute

118 infarction. These losses were considered to be within normal failure rates for this procedure.

119 Mice were evaluated using imaging as separate one-week and two-week post-infarction surgery

120 cohorts so that comparisons could be made with conventional ex vivo staining methods. Upon

121 completion of the imaging session, animals were never allowed to regain consciousness and

122 further induced with 4-5\% isoflurane anesthesia to ensure maximal anesthetic plane. Tissues

123 were then collected by cardiac excision for further analysis.

124

125 The mice were prepared for imaging by induction and maintenance with $4 \%$ isoflurane and 1.25 -

$1261.75 \%$ isoflurane in $100 \%$ oxygen, respectively. For anesthesia maintenance, the head was

127 placed in an anesthesia mask with a modified notched nosecone to allow access to the eye for

128 retro-orbital injection. A commercial depilatory cream was used to remove fur from the anterior

129 chest. Mice were placed in the supine position on a mobile heated imaging platform maintained

130 at $37^{\circ} \mathrm{C}$, with the extremities taped to electrocardiogram (ECG) electrodes for heart rate and

131 respiratory monitoring. Extreme care was taken to ensure that each mouse was at minimal

132 anesthetic depth to enable maximal physiological function. The ears were also taped to the

133 platform to decrease movement during the injection of microbubbles via the retro-orbital route.

135 A previously reported retro-orbital intravenous injection technique was used due to its proven

136 efficacy and ease of access (Wang et al. 2015a; Yardeni et al. 2011), but with further refinement.

137 A catheter was fashioned from a Terumo 0.5 inch $27 \mathrm{G}$ butterfly catheter by removing the

138 existing tubing and replacing it with $10 \mathrm{~cm}$ of polyethylene 20 tubing. After catheter assembly, 
139 and before introduction to the animal, flushing with $0.9 \%$ saline was performed to ensure the

140 smooth delivery of microbubbles without resistance to prevent unintended rupture upon delivery

141 to the mouse. The modified nosecone enabled visualization and access to the eye during catheter

142 placement and ear taping enabled stability to maintain catheter patency. Gentle pressure was

143 applied at the base of the skull to create mild protrusion of the eye. The needle was inserted with

144 the bevel towards the globe, into the medial canthus at approximately the lacrimal duct. The

145 catheter was then stabilized with tape and flushed with $50 \mu 1$ saline to test patency and venous

146 access. Once the retro-orbital catheter was placed, extreme care was taken to prevent movement

147 of the animal during subsequent imaging.

148

149 Microbubbles

150 The bolus perfusion model was chosen for this study due to the simplicity of the technique and

151 smaller total volumes of fluid delivered to the animal relative to the destruction-replenishment

152 model. After trials of various sizes of neutral size-isolated microbubbles (nSIMB) 5-8, 4-5, and

153 3-4 $\mu \mathrm{m}$, it was determined that the 3-4 $\mu \mathrm{m}$ SIMB (Advanced Microbubble Laboratories LLC,

154 Boulder, CO) provided an optimal enhancement of echogenicity. They were diluted to $4 \times 10^{8}$

155 bubbles $/ \mathrm{ml}$, for a total of $2 \times 10^{7}$ bubbles injected in a $50 \mu 1$ bolus, followed by a $50 \mu 1$ bolus

156 saline flush.

157

158 For comparison, we also tested size 3-4 $\mu \mathrm{m}$ cationic SIMB (cSIMB) due to their reported ability

159 to adhere to the negatively charged vascular endothelium (Sirsi et al. 2012). This was done to

160 attempt enhancement of the myocardium while the ventricular lumen was cleared of

161 microbubbles. These were administered in the same dilution as the nSIMB. 
163 Imaging Overview

164 We used the Vevo 2100 High-Resolution Micro-Ultrasound System and associated Vevo LAB

165 software v3.2 (FUJIFILM VisualSonics, Toronto, ON, Canada). All data was stored in the

166 central university maintained server, backed up twice daily. For comparison of our proposed

167 MCE technique with the commonly performed functional imaging methods used for mice, we 168 collected B-Mode and M-Mode parasternal long axis (PLAX) and parasternal short axis (PSAX)

169 images from each animal. For further comparison to MCE, the collected B-Mode images were

170 also evaluated using speckle tracking echocardiography (STE) for the functional evaluation of

171 cardiac strain. This software uses a Lagrangian model of strain (Theodoropoulos \& Xu 2008).

172

173 For MCE, we determined that the $21 \mathrm{MHz}$ MS250 linear array probe provided the best

174 combination of resolution and penetration. Additionally, this probe has the frequency necessary 175 to perform the NLC function and detection of the SIMB. A 3D motor controlled the probe and 176 created 3D images with a step size of $0.07 \mathrm{~mm}$ and a scan length of $10.06 \mathrm{~mm}$, recording 286

177 frames. These were gated to both respirations and the ECG. End-systole was chosen to acquire

178 images due to the more consistent signal intensity and the increased ability to visualize and trace

179 the myocardium (Verkaik et al. 2018). All images were stored for later evaluation. Image

180 analysis was performed by individuals blinded to the treatment (MI versus sham-operated) and

181 type of bubbles (nSIMB versus cSIMB).

182

183 Sequence of Image Acquisition 
184 Once the retro-orbital catheter was placed and the animal was prepared for imaging, we first 185 acquired standard 2D B-Mode and M-Mode PLAX images with the cursor placed at

186

187

188

189

190

191

192

193

194

195

196

197

198

199

200

201

202

203

204

205

206

approximately the mid-papillary level. The transducer was then turned to acquire PSAX images at the mid-papillary level in 2D B-Mode and M-Mode. Images were acquired with settings of frequency $21 \mathrm{MHz}$, power $100 \%$, and a dynamic range of $60 \mathrm{~dB}$. To ensure adequate inclusion of the entire heart we used a depth of $14 \mathrm{~mm}$ and a width of $14.04 \mathrm{~mm}$, which resulted in a frame rate of 167 frames/sec. M-Mode images were acquired with similar settings, except frame rate was 20 frames/sec, gate depth $12.92 \mathrm{~mm}$, and size $7 \mathrm{~mm}$. Time gain compensation controls were set to optimize image quality and recorded as a preset to be used with all imaging. After standard 2D B-Mode and M-Mode PLAX/PSAX images were acquired, the MCE data collection sequence was initiated. Before the delivery of microbubbles, a pre-scan was obtained. To do this, NLC Mode was initiated along with the simultaneous activation of 3D Mode (3D+NLC). During 3D+NLC Mode, gating was applied and a 3D pre-scan (i.e. pre-injection) of 286 images was acquired in the transverse PSAX plane along the entire length of the heart for later background subtraction. The settings in 3D Mode were $18 \mathrm{MHz}$, power $10 \%$, and dynamic range $40 \mathrm{~dB}$. Contrast and 2D gain were set at $24 \mathrm{~dB}$ and $21 \mathrm{~dB}$, respectively.

After completion of the $3 \mathrm{D}+\mathrm{NLC}$ pre-scan, the transducer was then rotated back to the sagittal plane to obtain a PLAX view and the machine was set to 2D NLC Mode to acquire the MCE arrival kinetic data from the initial microbubble injection. The timing of image acquisition was coordinated with the injection of the microbubbles so that an adequate baseline image and plateau of the kinetics of the microbubbles arriving could be recorded, $\sim 7$-seconds pre-injections, and $\sim 20$-seconds total. Prior to locking in the image acquisition regimen, we had tested 
207 microbubble stability by collecting additional post-injection PLAX 2D NLC images at $\sim 1-2$ min,

$208 \sim 8$-min, and 12-min post microbubble injection. From this, we determined that the stability of

209 circulating microbubbles had reached a plateau at $\sim 1-2$ min post-microbubble injection, were

210 still present at 8-min post-injection, but were largely cleared by $12-\mathrm{min}$. Therefore, to facilitate

211 comparison of nSIMB and cSIMB, another 2D NLC PLAX image was obtained at 8-min post-

212 injection. Additionally, to adequately capture the 3D+NLC post-scan while the microbubbles

213 were stable and still adequately in circulation, at $\sim 1-2$ minutes, we returned the probe to the

214 transverse PSAX and recorded the 3D+NLC post-scan. The collection of a 3D+NLC pre-scan

215 and post-scan facilitated background subtraction to be performed upon image analysis, allowing

216 quantification of the total microbubble percent agent (PA) of the myocardium while the

217 microbubbles were stably circulating.

218

219 After this entire protocol was completed with nSIMB, two high mechanical index burst

220 sequences were used to ensure full clearance of the animal of microbubbles. The entire protocol

221 above was then repeated in the same animal with the size 3-4 $\mu \mathrm{m}$ cSIMB. As explained, the 8-

$222 \min$ PLAX 2D NLC scan was utilized to compare the neutral versus cationic microbubble

223 myocardial lodging. None of the protocols described have been registered.

Image Analysis

226 Conventional Echocardiogram Assessments

227 M-Mode and 2D B-Mode images were loaded onto Vevo LAB 3.2 software. Anterior and

228 posterior LV walls of PLAX and PSAX M-Mode images were defined using the trace technique

229 and software algorithms calculated an ejection fraction (EF). VevoStrain software, which enables 
230 STE analysis from 2D B-Mode images, was used to calculate parameters including an additional

231 EF, global longitudinal strain (GLS), and global circumferential strain (GCS) using the 2D B-

232 Mode PLAX and PSAX images.

2D NLC Assessments

235 The kinetics of microbubble arrival as well as the stability of microbubbles at 8-min post-

236 injection was evaluated. 2D NLC microbubble arrival kinetics enabled evaluation of parameters

237 of relative blood perfusion and volume. The 8-min post-injection 2D NLC data was evaluated to

238 assess the stability of the nSIMB versus the cSIMB to determine if lodging of microbubbles in

239 the myocardium could be detected. To quantify these parameters, the 2D NLC video images,

240 which were collected with constant gain and dynamic range, were loaded into VevoCQ software

241 and images were corrected for motion artifact. A region of interest (ROI) was traced along with

242 the endo- and epicardium in PLAX so the whole 2D myocardium was included. The ROI was

243 traced while microbubbles were present in the myocardium, taking advantage of the enhanced

244 delineation of the endocardium. From these 2D NLC videos, the software calculated a wash-in-

245 rate (WiR) representing relative blood perfusion, and peak enhancement (PE) representing

246 relative blood volume present in the myocardium (Greis 2011). The software calculated these

247 from the maximum slope and plateau of the microbubble arrival kinetics to the myocardium,

248 respectively (VisualSonics) (Fig. 1). For cSIMB and nSIMB comparisons, the ROI was copied

249 and pasted onto the 8-min image to ensure that same area of the myocardium was under

250 consideration and to further understand the characteristics of the differentially charged

251 microbubbles through this time frame. 
253 3D PA Assessments

254 3D PA data is obtained from a series of $\sim 300$ individual 2D image frames that are compiled to

255 form a 3D image. 3D scan collection time typically took less than three minutes. Microbubbles

256 were likely stably circulating during the scan as we showed that microbubbles were present for at

257 least eight minutes (Table 1). To facilitate analysis of the 3D+NLC data to calculate the PA, the

258 pre-scan and $\sim 1-2-$ min post-scan 3D+NLC images were loaded into the Vevo LAB 3D software.

259 The ROIs were traced in PSAX on the 1 -2-min post-scan image over the epicardium and

260 endocardium, so the entire 3D myocardium was included, using the multi-slice tracing technique

261 (Fig. 2). ROIs were traced individually, every 5-10 frames, from the base to the apex of the heart

262 to provide a smooth transition from each tracing. The 3D+NLC pre-scan (pre-injection) images

263 were used as background subtraction from the post-scan (post-injection) images to calculate a

264 percent agent (PA). This was calculated from the number of pixels within a defined volume,

265 which are associated with the non-linear contrast signal given off by microbubbles. The PA

266 indicates the relative vascularity of the entire defined myocardium in 3D.

267

268 TTC Evaluation

269 For TTC staining, the ex vivo myocardium was sliced into four sections in the transverse plane

270 for comparison to the MCE parameters. TTC was used due to its long history as the gold

271 standard of quantifying MI ex vivo (Fishbein et al. 1981; Klein et al. 1981). It was also used as

272 the sole staining technique for simplicity purposes and because it indirectly indicates perfusion in

273 a permanent coronary ligation model. Within an hour of completion of ultrasound imaging, the

274 mice were deeply sedated using $4-5 \%$ isoflurane in $100 \%$ oxygen and the heart was excised. The

275 heart was placed in a solution containing $56 \mathrm{mg}$ of heparin/L in phosphate buffered saline and 
276 allowed to pump thoroughly. The aorta of the heart was cannulated with a $24 \mathrm{G}$ mouse gavage

277 needle cannula and tied in place with 6-0 silk suture (Bohl et al. 2009). The heart was then gently

278 flushed with 2-3 $\mathrm{ml}$ of the phosphate buffered saline/heparin, taking care to ensure that the

279 vasculature of the heart was effectively cannulated and not the ventricular lumen. Using a

280 syringe pump, the heart was flushed at $67 \mu \mathrm{l} / \mathrm{min}$ for $15 \mathrm{~min}$ with $1 \%$ TTC solution $(0.1 \mathrm{~g} / 10 \mathrm{ml}$

281 per vial) (Sigma Aldrich, \#17779). The heart was cut from the cannula, atria removed, and

282 placed in $\sim 5-10 \mathrm{ml}$ of formalin. After a minimum of 2 hours, the heart was sliced into 5 equal

283 sections using a Zivic Heart Slicer and returned to the formalin for 24 hours before photographs

284 were taken.

285

286 The stained heart sections were then photographed using the Leica S6D microscope and EC3

287 camera and associated LAS v4.12 software (Leica Microsystems, Buffalo Grove, IL). Percent

288 infarction was calculated by dividing the area of infarcted myocardium (white area) by the total

289 area of myocardium (white+bright red area). The sum of these areas from each slice was used so

290 that the reported percentage would best represent the amount of infarction of the entire left

291 ventricle, enabling comparison of this value with the 3D PA.

292

293 Statistics

294 All statistics were analyzed using GraphPad Prism ${ }^{\circledR}$ version 7.0 (GraphPad Software, USA).

295 Power calculations to determine sample size were performed based on change in EF of MI

296 versus sham-operated animals of surgeries performed previously in our hands. Using an alpha of

2970.05 with $95 \%$ power, power calculation revealed that an $\mathrm{n}$ of 2 animals per group were

298 sufficient to detect statistical significance. However, we utilized an n of 4-6 per group/cohort. 
299 Each conventional and MCE imaging technique was used to compare the MI animals to the

300 sham-operated animals using multiple t-tests. Pearson correlations between each MCE parameter

301 and the TTC histological standard were made with linear regression. Sham-operated and MI

302 animals in their respective time cohort were combined in the correlation graphs to assess how the

303 technique correlates with TTC staining in a range of degree of MI. A (two-tailed) $P$ value of less

304 than 0.05 was considered significant and all data are represented as mean \pm standard deviation.

305 For the data represented in Fig. 1-4, animals which failed to have infarctions as determined by

306 TTC staining were removed from mean calculations. These scatter plots include the failed MIs as

307 a green data point to assess if each technique could differentiate them from the rest of the MI

308 cohort.

309

310 Results

311 Animal Model and Injection Technique

312 Standard LAD ligation surgery to induce MI was utilized to test the proposed MCE imaging

313 methods. The LAD ligation surgery resulted in a 71\% survival of animals, giving a total of 20

314 mice included in this study, resulting in $n=4-6$ animals per group and cohort. One animal in the

315 1-week cohort failed to have an MI and one animal in the 2-week cohort had a very mild MI as

316 determined by TTC staining (Fig. 3C and D). These failed MIs were therefore removed from

317 comparison of MI to sham-operated animals in terms of mean data analysis in Fig. 1-4. However,

318 note that the two failed MI animals were included in correlation calculations and graphical

319 analyses to demonstrate the ability of each MCE parameter to correlate with TTC over a wide

320 range of infarction sizes. The retro-orbital injection was performed successfully in all animals 
321 and with relative ease after mastery of the technique. There was damage to the globe of the eye

322 in only one animal, upon our first attempt of the injection technique.

323

324 TTC Staining Results

325 TTC staining of the myocardium was performed in the 1-week and 2-week cohorts at endpoint to

326 enable comparison of this gold-standard infarction quantification method with the proposed

327 MCE parameter. TTC staining of the myocardium was successful in all except one mouse which

328 was excluded in all statistics. Fig. 3A and B are representative images of TTC stained sham-

329 operated and MI hearts. Fig. 3E depicts the mean values of the percent infarct of the total

330 myocardium (TTC\% infarction). The two failed MIs and the failed TTC stained heart were

331 removed from these mean calculations. For the 1-week cohort, MI $(n=4)$ and sham-operated

332 animals $(\mathrm{n}=4)$ resulted in a TTC $\%$ infarction of $31.64 \pm 4.04 \%$ and $1.53 \pm 1.80 \%(P<0.001)$,

333 respectively. For the 2 -week cohort, MI $(n=4)$ and sham-operated animals $(n=5)$ resulted in a

334 TTC percent infarction of $22.04 \pm 5.6 \%$ and $2.30 \pm 4.24 \%(P<0.001)$, respectively. A scatter plot

335 of these data, which include and indicate the failed MIs as a green color, is seen in Fig. 3E.

336

337 Conventional Echocardiogram Results

338 Echocardiograms to collect conventional functional data were performed in the 1-week and 2-

339 week cohorts to further compare to the proposed MCE technique parameters. Echocardiographic

340 data of M-mode EF is depicted in Fig. 4. Again, the two failed MIs were excluded from mean

341 calculations giving $n=4$ of the sham-operated cohort and $n=5$ of the MI cohort. In the 1 -week

342 cohort, MI and sham-operated animal M-Mode EFs were $26.05 \pm 12.89 \%$ and $60.86 \pm 5.86 \%$

$343(P=0.002)$, respectively. For the 2-week cohort, MI and sham-operated animal M-Mode EFs 
344 were $23.20 \pm 12.66 \%$ and $61.87 \pm 9.06 \%(P=0.001)$, respectively. A M-Mode EF scatterplot is

345 represented in Fig. 4A and the failed MIs are included in this figure. Fig. 4B and C represent

346 strain analysis data of GLS and GCS, again with the failed MIs included in the scatterplot. Note

347 how M-Mode EF and GCS could not specify the failed MIs; however, GLS measurements

348 identified both failed MIs as indicated with arrows. The mean data from these measurements are

349 seen in Fig. 4 with the failed MIs excluded from the means.

350

351

MCE Parameters

352 Image quality was good and remained reasonably consistent with all MCE images after optimal

353 presets were determined (Fig. 1). PE, WiR, and PA mean values using nSIMB with standard

354 deviations are seen in Fig. 1 and 2.

355

356

The 2D NLC PE and WiR represent data from the initial microbubble kinetic arrival period with nSIMB and are depicted in Fig. 1. These parameters reflect relative blood volume (PE) and relative blood perfusion (WiR) of the myocardium. For the 1-week cohort, the PE for MI and

359 sham-operated animals were $75.08 \pm 27.23$ and $442.29 \pm 163.00(P=0.002)$, respectively. For the $2-$

360 week cohort, the PE for MI and sham-operated animals were $65.68 \pm 23.61$ and $270.39 \pm 75.01$

$361(P<0.001)$, respectively. For the 1-week cohort, the WiR for MI and sham-operated animals

362 were $70.32 \pm 95.91$ and $401.86 \pm 140.44(P=0.004)$, respectively. For the 2-week cohort, the WiR

363 for MI and sham-operated animals were $39.27 \pm 26.07$ and $111.30 \pm 65.12(P=0.056)$, respectively.

364 Fig. $1 \mathrm{G}$ and $\mathrm{H}$ depict the WiR and PE scatterplots with the failed MIs included and indicated as a 365 green color. Note that for the WiR, the failed MI could be detected in the 1-week cohort, but not 
366 in the 2-week cohort. However, for PE, the failed MI was undetectable in the 1-week cohort but

367 was detectable in the 2-week cohort.

368

369 The 3D PA technique is proposed to provide a more complete picture of vascularity since it

370 includes the microbubbles circulating in the entire myocardium, rather than just a $2 \mathrm{D}$ image

371 plane as is collected with PE and WiR. The final PA value was calculated by performing a

372 background subtraction of the pre-scan (pre-injection) image from the $\sim 1-2$-min post-scan (post-

373 injection) image while the nSIMB were stably circulating. Fig. 2A shows an example image of

374 the ROI of the myocardial space defined to perform the 3D reconstruction. The PA was

375 determined from this 3D ROI and quantifies the total amount of circulating microbubbles within

376 the entire myocardium. As shown in Fig. 2, the PA for the 1-week cohort for MI and sham-

377 operated mice were $46.32 \pm 11.70 \%$ and $73.27 \pm 10.02 \%(P=0.008)$, respectively. The PA for the

378 2-week cohort for MI and sham-operated mice were $42.17 \pm 6.34 \%$ and $81.89 \pm 9.49 \%$

$379(P<0.001)$, respectively. Again, the failed MIs were excluded from these calculations. However, 380 as can be seen in Fig. 2B, the PA MCE technique was able to detect both the failed MIs in both 381 cohorts as indicated by the arrows.

MCE Correlations with Conventional Techniques

To further validate the proposed MCE parameters with conventionally accepted MI evaluation methods, the resultant data was compared using liner regression. Pearson Correlations were 386 plotted for TTC\% infarction against each of the MCE parameters (PE, WiR, and PA) and for each cohort as shown in Fig. 5. Data from failed MI's were included in correlations. A 1-week 
389 parameters were excluded, resulting in $n=4$ in both of the sham-operated groups, $n=5$ in the 1390 week MI group, and $\mathrm{n}=6$ in the 2-week MI group. The calculated $\mathrm{r}$ - and $P$ values are shown in

391 Fig. 5. Correlations of TTC\% infarction versus PE in the 1 -week cohort resulted in an $\mathrm{r}=-0.74$

392 (CI -0.94 to 0.14) and in the 2-week cohort resulted in $\mathrm{r}=-0.76$ (CI -0.94 to 0.24). Correlations

393 of TTC\% infarction versus WiR in the 1 -week cohort resulted in $r=-0.68$ (CI -0.93 to 0.025)

394 and in the 2 -week cohort $r=-0.50$ (CI -0.86 to 0.19$)$. Correlations of TTC $\%$ infarction versus PA

395 in the 1 -week cohort resulted in $\mathrm{r}=-0.78(\mathrm{CI}-0.95$ to 0.24$)$ and in the 2 -week cohort $\mathrm{r}=-0.77$

396 (CI -0.94 to 0.26$)$. The only insignificant correlation was the WiR 2-week cohort $(P=0.14)$.

397

398 The three MCE parameters of PE, WiR, and PA were also correlated with the conventional

399 functional parameters of 2D EF and GLS measured in PLAX. These results are seen in Table 2.

400 All animals were included in these correlations giving an $n=4$ in the sham-operated group and

$401 \mathrm{n}=6$ in the MI group in both 1-week and 2-week cohorts. As before, all correlations represent

402 strong relationships between the MCE parameters and the conventional functional parameters,

403 with the exception of the WiR, which only correlated significantly with the 2D EF 1-week cohort

$404(P=0.036)$.

405

406 Cationic vs Neutral Microbubbles

407 As stated, we performed the entire protocol in each animal with both nSIMB and cSIMB. The

408 hypothesis was that the cSIMB would lodge in the myocardium vessels and enable improved

409 imaging and quantification. Since the PE is the point at which the microbubbles have reached a

410 stable plateau in circulation, it is proposed that if the bubbles were lodging in the myocardium

411 successfully, then the PE value would be higher at the 8-min timepoint in the cSIMB than the 
412 nSIMB. To address this hypothesis, only sham-operated animals were included and 1- and 2-

413 week cohorts were combined for $n=8$ for each cohort of nSIMB or cSIMB microbubble type.

414 Table 1 depicts the PE value from the initial microbubble injection (PE Arrival) versus the PE at

415 8-min (PE 8-min) for both nSIMB and cSIMB. The PE at the plateau after initial injection (PE

416 Arrival) was $356.34 \pm 149.14$ for the nSIMB and $354.58 \pm 185.17$ for the cSIMB with a $P$ value of

417 0.98, indicating no difference in lodging. After 8-min of plateau (PE 8-min), the PE was

$41849.35 \pm 47.52$ for the nSIMB and $24.64 \pm 19.28$ for the cSIMB calculating a $P$ value of 0.19 .

419 Although insignificant, this raises the possibility that there may have even been reduced lodging

420 of the cSIMB. The reduction of cSIMB from $354.58 \pm 185.17$ at initial plateau to $24.64 \pm 19.28$ at

4218 -min plateau certainly provides no evidence of lodging of the cationic microbubbles. The WiR

422 value for the initial microbubble injection arrival kinetics for each SIMB type is also included in

423 Table 1, however, the differences in these values for the two types of microbubbles exhibited

424 excess variability.

\section{Discussion}

427 This study provides proof of concept for a complementary technique to conventional TTC staining and echocardiography using noninvasive myocardial perfusion parameters. While these

NLC tools have been primarily developed for the evaluation of the hemodynamic characteristics of tumors, we propose that they have applicability for evaluation of the heart. Overall, we successfully induced infarctions in all the mice assigned to the MI group, except two. Of these two fails, one did not exhibit any infarction and the other had an extremely mild MI. These two failed MIs were detected via TTC staining and conventional functional imaging techniques. We

434 then tested multiple MCE parameters collected using NLC that were able to successfully 
435 differentiate sham-operated vs MI animals. Importantly, these parameters correlated well with

436 the gold standard TTC and conventional ultrasound functional imaging. Specifically, the 3D PA

437 was able to detect both failed MIs and was well correlated with TTC at each timepoint. PE and

438 WiR each detected one failed infarction. 2D B-Mode EF and GLS were also able to detect both

439 failed MIs, however the commonly utilized, M-Mode EF and GCS were not.

440

441 The 3D PA value is thought to give information regarding the total vascularity of the

442 myocardium as it indicates the percent of contrast agent present in the user-defined 3D region of

443 interest. This is the parameter that most resembles the TTC technique as they are both 3D and

444 derived from the perfusion of the myocardium. Just as TTC is less likely to stain the infarcted

445 myocardium (Klein et al. 1981), the contrast agent is less likely to be present in the infarcted

446 myocardium, resulting in less overall NLC signal transmitted from the microbubbles. In light of

447 this, we advocate that the proposed MCE PA parameter can serve as a kind of "in vivo TTC."

448 However, PA adds the benefit of providing in vivo perfusion data and can likely be used

449 repeatedly throughout the experimental paradigm. Obvious value can be seen for this in models

450 in which treatments of MI may be detected while in vivo at intermediate experimental

451 timepoints, but otherwise missed at the endpoint. Additionally, this method may have utility in

452 studies involving genetic manipulation of animals in which genes may be turned off and on, 453 especially if the microvasculature is influenced. However, future studies will need to address

454 whether NLC MCE imaging of the myocardium will be sensitive enough for subtler models of 455 heart disease. 
457 The PE parameter was statistically significant in differentiating sham-operated vs MI animals at

458 both time points and correlated well with conventional methods. The WiR was statistically

459 significant in its ability to differentiate sham-operated vs MI animals and correlated with TTC

460 staining with the 1-week cohort, but not with the 2-week cohort. With our study's findings of the

461 MCE parameters differentiating sham-operated vs MI animals and correlating significantly with

462 gold standards, it is proposed that these values can provide valuable additional hemodynamic

463 information regarding the perfusion of the myocardium. We speculate in our study that the PE

464 value represents the relative blood volume within the myocardium. Our findings indicate this as

465 lower PE values were observed in animals with more severe myocardial infarctions resulting in

466 less perfusion and lower number of microbubbles present within the myocardium to emit a NLC

467 signal. Myocardial blood volume has been shown to be useful to the researcher, correlating with

468 functional aspects of coronary artery disease such as myocardial oxygen consumption

469 (McCommis et al. 2010). The area under the curve value was also calculated by the software and

470 could be considered; however, PE was chosen for this study as area under the curve best

471 represents vascularity in tissue that has complex feeding vessels, such as tumors (Greis 2011).

472 The PE value is limited to providing information in a 2D plane; however, it provides the

473 advantage of giving relative blood volume from the total NLC signal in that 2D image.

475 The WiR parameter resulted in the least amount of significant data of the MCE parameters,

476 likely due to variation in microbubble injection rate. It needs to be investigated further to

477 determine its value, given these results. Since WiR represents the rate of blood entering the

478 myocardium, it is assumed to indicate the perfusion of the myocardium. This is one of the key

479 advantages of MCE because it can provide information about micro-vessels and collateral vessel 
480 development, which is lost in other imaging techniques (Kaul 2008). Myocardial perfusion with

481 other modalities has an established role in research with calculating myocardial flow reserve,

482 enhancing the researcher's ability to assess coronary artery disease (McCommis et al. 2010). The

483 destruction-reperfusion technique may provide more accurate initial myocardial perfusion data

484 data as it eliminates the effects of the microbubbles having to traverse from the injection site to

485 the myocardium (Greis 2011). However, the bolus technique was chosen due to the lower

486 volumes required for microbubble administration and increased ease for the researcher. Mice

487 with MI commonly exhibit heart failure which would be further exacerbated by the volumes

488 required from continuous infusions. Also, the ease of the bolus technique makes it more efficient

489 for researchers to implement as it doesn't require an infusion pump and has been shown to have

490 a higher contrast signal-to-noise ratio (Averkiou et al. 2020). Perhaps in mouse models where the

491 systolic function is largely preserved, the destruction-reperfusion technique could be used to

492 provide more accurate $\mathrm{WiR}$ data.

493

494 Our findings regarding M-Mode EF are consistent with previous studies that have compared 495 these techniques and found limitations for the use of M-Mode in MI (Chen et al. 2016). M-Mode

496 has the advantage of being fast and relatively easy to perform and may be used to assess

497 ventricular function when studying non-coronary artery disease or animals that do not have

498 regional wall motion abnormalities. Still, M-Mode assessment and the use of the Teichholz

499 formula to calculate EF in mice are widely utilized (Benavides-Vallve et al. 2012). However, our

500 observed limitations with M-Mode in assessing animals with MIs likely reflects why it is not

501 recommended in clinical practice (Lang et al. 2006). The 2D B-Mode EF parameter is much

502 more likely to account for the infarcted portion of the heart. The accuracy of our GLS 
503 measurements to detect failed MIs reflects previously validated STE imaging in mice (Bauer et

504 al. 2011). GLS in clinical practice is continuing to develop and is currently recommended with

505 patients receiving chemotherapy (Plana et al. 2014; Smiseth et al. 2016). The inability of the

506 GCS measurement to detect the failed/very mild MIs likely reflects the same limitation seen with

507 M-Mode in that it is assessing a single PSAX plane that may miss analyzing a more distal

508 infarcted region. Although STE will likely gain utility and M-Mode may continue to have an

509 application, these techniques are still limited.

510

511 The retro-orbital catheter injection technique is a valuable method for injecting microbubbles.

512 Researchers often use the lateral tail vein for microbubble injections which can be difficult to

513 perform in anesthetized animals exhibiting peripheral vasoconstriction or those with dark tail-

514 colors. Retro-orbital injections are relatively easy to perform and shown to deliver the injected

515 substance with the same kinetics as if it were injected in the tail vein (Wang et al. 2015a;

516 Yardeni et al. 2011). Our modifications to the catheter, as described in the methods section,

517 allowed us to keep the catheter inserted in the retro-orbital sinus to facilitate multiple injections.

518 We perforated one mouse eye during the initial attempt; however, all subsequent injections were

519 performed successfully and efficiently by a novice injector.

521 We initially attempted to lodge neutral microbubbles within the myocardial vasculature as

522 previously demonstrated (Kaufmann et al. 2007), with the goal of being able to visibly identify

523 segmental perfusion defects without shadowing from microbubbles present in the ventricular

524 lumen. Therefore, we trialed nSIMB 3-4, 4-5, and 5-8 $\mu \mathrm{m}$ from the manufacturer, hypothesizing

525 that the larger bubbles may lodge. However, while the data is not presented here, we were not 
526 able to reproduce the lodging of nSIMBs in the myocardium with a ventricular lumen clear of

527 microbubbles. Reasons for this may include differences in the composition of the microbubbles

528 (i.e. different manufacturers) or differences in technique from previous publications. We also

529 attempted to perform this lodging with cationic microbubbles, cSIMB 3-4 $\mu \mathrm{m}$, due to previous

530 reports that the cation charge may adhere to negatively charged endothelium (Sirsi et al. 2012).

531 Our data indicated no significant evidence of lodging of cationic microbubbles. We were also

532 unable to detect differences between nSIMB and cSIMB using the kinetic parameters seen in

533 Table 1. However, the cSIMB measurements were performed after the nSIMB, possibly resulting

534 in shifting of the animal or cardiac structure and more error with these measurements.

536 We determined that the nSIMB 3-4 $\mu$ m gave us the best signal and after several trials to find the 537 optimal microbubble concentration, we were able to improve the quality of images with reduced 538 shadowing artifact from ventricular lumen microbubbles. Shadowing artifacts are still a 539 limitation of the MCE technique, including those caused by the rib and lung, common to all 540 mouse echocardiograms. Shadowing can also result from the contrast present in the tissue

541 surrounding the heart along with potential scar tissue in the chest wall from MI surgery. Finally,

542 the pericardium was noted to intermittently produce artifact that may have been detected as a 543 false NLC signal, possibly reducing the accuracy of the WiR parameter.

545 A limitation often described with MCE is the lack of harmonic imaging associated with the high

546 frequencies required for imaging mice. We noted a better signal with the size 3-4 $\mu \mathrm{m}$

547 microbubbles and this may be attributed to smaller bubbles producing more NLC signal with

548 higher frequency transducers (Goertz et al. 2003). Although harmonic imaging may be reduced 
549 at these frequencies, we were able to detect enough NLC signal to perform these various MCE

550 parameters, which has also been described elsewhere (Raher et al. 2007). This NLC imaging

551 provides an advantage by filtering out other "noise" in the myocardium so that only

552 microbubbles present in the vasculature is detected, giving a more accurate assessment of 553 perfusion.

554

555 Other studies have described various techniques for quantifying myocardial perfusion/MI in

556 mice (Alvarez et al. 2018; French et al. 2006), but they do not quantify the NLC signal itself nor

557 use readily available equipment and software as presented here. Rather, their measurements

558 solely utilized pixel intensity and specialized software that may not be readily available or add

559 complexity to a researcher's practice. Our study also improved on these by offering multiple

560 time points and comparing the techniques to TTC staining and conventional functional

561 measurements.

562

563 While the power calculation indicated adequate sample size for statistical power, a major

564 limitation of the present study includes the small sample size. Comparisons of the presented

565 methods could be affected by variance in body weight, heart rate, and respiratory rate which may

566 alter the metabolism of the microbubbles. The anesthetic regimen should be chosen and

567 monitored carefully considering its effects of cardiovascular physiology and reported influence

568 on echocardiographic parameters (Wang et al. 2015b). However, isoflurane anesthesia is the

569 most widely used anesthetic for evaluation of mouse echocardiogram and thus was chosen in this

570 study. Other limitations include that the user who performed the scans was new to mouse

571 echocardiography and likely more prone to errors such as apical foreshortening when scanning. 
572 However, this may be considered an advantage that a novice user can readily perform this

573 technique. The technique and measurements may have been performed with more accuracy as

574 the user improved. When injecting the microbubbles, resistance can cause microbubbles to burst

575 while in the syringe, altering the concentration of microbubbles actually injected. We addressed

576 this by taking extreme care that the catheter once made was free moving by testing it with saline.

577 Finally, in terms of logistics, gating for the heart rate in severely infarcted animals without

578 obvious QRS complex occasionally failed to detect the ECG signal, resulting in movement of the 579 heart and a displaced ROI.

580

581 Perhaps the largest limitation of this study was the inability to visibly distinguish infarcted from

582 non-infarcted zones on 2D echocardiography. This may be due to inadequate resolution of the 583 small mouse myocardium, especially after excessive thinning of the myocardial wall post

584 infarction. Without this direct visualization of the perfusion defect, whether the tested parameters 585 truly reflect infarction will need further study. It may be worth considering that new

586 developments into our understanding of angiogenesis suggests that perfusion in MI zones may

587 not be completely absent (Kobayashi et al. 2017). Perhaps with newer technology, including

588 newer versions of ultrasound hardware and software, this resolution could be improved allowing

589 segmental perfusion analysis and better characterization of the perfusion in the border of, and

590 within, the infarction. Additionally, a report of the utility of the NLC system for the evaluation of

591 the myocardium may spark interest in the development of software expressly for the purpose.

592 Magnetic resonance imaging provides many advantages over echocardiography regarding

593 cardiac anatomy resolution and soft tissue analysis; however, this modality is not widely 
594 accessible to researchers currently and continues to have limitations due to the high heart rate 595 and respiratory rate of a mouse (Bakermans et al. 2015).

596

597 Conclusion

598 Our study evaluates the ability of various MCE kinetic parameters to identify and characterize $599 \mathrm{MI}$ in an in vivo mouse model. Using readily available equipment and software in concert with 600 the most commonly utilized anesthetic and cardiovascular assessment methods, MCE enabled 601 collection of additional data to add value to the researcher toolbox. The majority of the PE, WiR, 602 and 3D PA parameters correlated well with TTC staining and conventional functional 603 echocardiography measures in 1-week and 2-week post-MI cohorts. Our findings suggest that 604 these parameters may provide complementary perfusion/hemodynamic information to the 605 functional parameters already established with standard echocardiograms. However, further 606 investigation of MCE in mouse models of MI is required before widespread adoption of this 607 technique into basic scientific research. This study provides the groundwork for further 608 assessment of these parameters to determine their accuracy and role in the evaluation of murine 609 MI.

610

\section{Author Contributions}

612 Author \#1 performed all echocardiography, retro-orbital catheter placement, microbubble 613 injection, and PE, WiR, PA, and M-Mode measurements blinded to which animals were 614 infarcted or sham-operated. Author \#2 performed all STE and 2D EF measurements blinded to 615 which animals were MI or sham-operated. Author \#3 performed all LAD ligations, TTC staining 
616 and photography, and TTC measurements. Authors \#1 and \#3 were involved in writing this

617 paper.

618

619 Acknowledgements

620 We would like to thank Dr. Doug Martin and Dr. Yi-Fan Li (University of South Dakota) for

621 their assistance with this project and the preparation of this manuscript. We would also like to

622 thank Jerad Schlobohm (South Dakota State University) for his help preparing the figures.

623 Lastly, we would like to express appreciation for access to the equipment and services provided

624 by the Physiology Core Facility in the Department of Basic Biomedical Sciences at The

625 University of South Dakota.

626

627

628

629

630

631

632

633

634

635

636

637

638

639

640

641

642

643

644

645

646

647

648

649

650

651

652

\section{References}

2,3,5-Triphenyl-tetrazolium chloride solution, for microbiology. Available at https://pubchem.ncbi.nlm.nih.gov/substance/329751567.

Ahn D, Cheng L, Moon C, Spurgeon H, Lakatta EG, and Talan MI. 2004. Induction of myocardial infarcts of a predictable size and location by branch pattern probability-assisted coronary ligation in C57BL/6 mice. American Journal of Physiology-Heart and Circulatory Physiology 286:H1201H1207. 10.1152/ajpheart.00862.2003

Alvarez E, Dalton ND, Gu Y, Smith D, Luong A, Hoshijima M, Peterson KL, and Rychak J. 2018. A novel method for quantitative myocardial contrast echocardiography in mice. American Journal of Physiology-Heart and Circulatory Physiology 314:H370-H379. 10.1152/ajpheart.00568.2017

Averkiou MA, Bruce MF, Powers JE, Sheeran PS, and Burns PN. 2020. Imaging Methods for Ultrasound Contrast Agents. Ultrasound in Medicine and Biology 46:498-517. 10.1016/j.ultrasmedbio.2019.11.004

Bakermans AJ, Abdurrachim D, Moonen RP, Motaal AG, Prompers JJ, Strijkers GJ, Vandoorne K, and Nicolay K. 2015. Small animal cardiovascular MR imaging and spectroscopy. Prog Nucl Magn Reson Spectrosc 88-89:1-47. 10.1016/j.pnmrs.2015.03.001

Bauer M, Cheng S, Jain M, Ngoy S, Theodoropoulos C, Trujillo A, Lin F-C, and Liao R. 2011. Echocardiographic speckle-tracking based strain imaging for rapid cardiovascular phenotyping in mice. Circulation research 108:908-916. 10.1161/CIRCRESAHA.110.239574

Benavides-Vallve C, Corbacho D, Iglesias-Garcia O, Pelacho B, Albiasu E, Castaño S, Muñoz-Barrutia A, Prosper F, and Ortiz-de-Solorzano C. 2012. New Strategies for Echocardiographic Evaluation of Left Ventricular Function in a Mouse Model of Long-Term Myocardial Infarction. PLOS ONE 7:19. 10.1371/journal.pone.0041691

Bhan A, Sirker A, Zhang J, Protti A, Catibog N, Driver W, Botnar R, Monaghan MJ, and Shah AM. 2014. High-frequency speckle tracking echocardiography in the assessment of left ventricular function 
653

654

655

656

657

658

659

660

661

662

663

664

665

666

667

668

669

670

671

672

673

674

675

676

677

678

679

680

681

682

683

684

685

686

687

688

689

690

691

692

693

694

695

696

697

698

699 and remodeling after murine myocardial infarction. American Journal of Physiology-Heart and Circulatory Physiology 306:H1371-H1383. 10.1152/ajpheart.00553.2013

Bohl S, Medway DJ, Schulz-Menger J, Schneider JE, Neubauer S, and Lygate CA. 2009. Refined approach for quantification of in vivo ischemia-reperfusion injury in the mouse heart. American Journal of Physiology-Heart and Circulatory Physiology 297:H2054-H2058. 10.1152/ajpheart.00836.2009

Chen J, Hammoudi N, Benard L, Ceholski DK, Zhang S, Lebeche D, and Hajjar RJ. 2016. The Probability of Inconstancy in Assessment of Cardiac Function Post-Myocardial Infarction in Mice. Cardiovascular pharmacology: open access 5:195. 10.4172/2329-6607.1000195

Chen X, Cui K, Xiu J, Lin H, Lao Y, Zhou B, Liang F, Zha D, Bin J, and Liu Y. 2009. Evaluation and simplified measurement of infarct size by myocardial contrast echocardiography in a rat model of myocardial infarction. The International Journal of Cardiovascular Imaging 25:713-716. 10.1007/s10554-009-9474-x

Dourado PMM, Tsutsui JM, Mathias Jr. W, Andrade JL, da Luz PL, and Chagas ACP. 2003. Evaluation of stunned and infarcted canine myocardium by real time myocardial contrast echocardiography. Brazilian Journal of Medical and Biological Research 36:1501-1509.

Fishbein MC, Meerbaum S, Rit J, Lando U, Kanmatsuse K, Mercier JC, Corday E, and Ganz W. 1981. Early phase acute myocardial infarct size quantification: Validation of the triphenyl tetrazolium chloride tissue enzyme staining technique. American Heart Journal 101:593-600. https://doi.org/10.1016/0002-8703(81)90226-X

French BA, Li Y, Klibanov AL, Yang Z, and Hossack JA. 2006. 3D perfusion mapping in post-infarct mice using myocardial contrast echocardiography. Ultrasound in Medicine and Biology 32:805-815. 10.1016/j.ultrasmedbio.2006.03.002

Goertz DE, Frijlink M, Bouakaz A, Chin CT, Jong Nd, and Steen AWFvd. 2003. The effects of bubble size on nonlinear scattering from microbubbles. IEEE Symposium on Ultrasonics, 2003. p 1503-1506 Vol.1502.

Greis C. 2011. Quantitative evaluation of microvascular blood flow by contrast-enhanced ultrasound (CEUS). Clinical Hemorheology and Microcirculation 49:137-149. 10.3233/CH-2011-1464

Kaufmann BA, Lankford M, Behm CZ, French BA, Klibanov AL, Xu Y, and Lindner JR. 2007. High-resolution Myocardial Perfusion Imaging in Mice with High-frequency Echocardiographic Detection of a Depot Contrast Agent. Journal of the American Society of Echocardiography 20:136-143. 10.1016/j.echo.2006.08.008

Kaul S. 2008. Myocardial Contrast Echocardiography. Circulation 118:291-308. doi:10.1161/CIRCULATIONAHA.107.747303

Klein HH, Puschmann S, Schaper J, and Schaper W. 1981. The mechanism of the tetrazolium reaction in identifying experimental myocardial infarction. Virchows Archiv A 393:287-297. 10.1007/BF00430828

Kloner RA. 2020. Stunned and Hibernating Myocardium: Where Are We Nearly 4 Decades Later? Journal of the American Heart Association 9:e015502. doi:10.1161/JAHA.119.015502

Kobayashi K, Maeda K, Takefuji M, Kikuchi R, Morishita Y, Hirashima M, and Murohara T. 2017. Dynamics of angiogenesis in ischemic areas of the infarcted heart. Scientific Reports 7:7156. 10.1038/s41598-017-07524-x

Lang RM, Bierig M, Devereux RB, Flachskampf FA, Foster E, Pellikka PA, Picard MH, Roman MJ, Seward J, Shanewise J, Solomon S, Spencer KT, St. John Sutton M, and Stewart W. 2006. Recommendations for chamber quantification *. European Journal of Echocardiography 7:79108. 10.1016/j.euje.2005.12.014

McCommis KS, Goldstein TA, Abendschein DR, Misselwitz B, Pilgram T, Gropler RJ, and Zheng J. 2010. Roles of myocardial blood volume and flow in coronary artery disease: an experimental MRI 
700

701

702

703

704

705

706

707

708

709

710

711

712

713

714

715

716

717

718

719

720

721

722

723

724

725

726

727

728

729

730

731

732

733

734

735

736

737

738

739

740

741

742

743

744

745

study at rest and during hyperemia. European radiology 20:2005-2012. 10.1007/s00330-0101740-8

Michael LH, Entman ML, Hartley CJ, Youker KA, Zhu J, Hall SR, Hawkins HK, Berens K, and Ballantyne CM. 1995. Myocardial ischemia and reperfusion: a murine model. American Journal of PhysiologyHeart and Circulatory Physiology 269:H2147-H2154. 10.1152/ajpheart.1995.269.6.H2147

Plana JC, Galderisi M, Barac A, Ewer MS, Ky B, Scherrer-Crosbie M, Ganame J, Sebag IA, Agler DA, Badano LP, Banchs J, Cardinale D, Carver J, Cerqueira M, DeCara JM, Edvardsen T, Flamm SD, Force T, Griffin BP, Jerusalem G, Liu JE, Magalhães A, Marwick T, Sanchez LY, Sicari R, Villarraga HR, and Lancellotti P. 2014. Expert Consensus for Multimodality Imaging Evaluation of Adult Patients during and after Cancer Therapy: A Report from the American Society of Echocardiography and the European Association of Cardiovascular Imaging. Journal of the American Society of Echocardiography 27:911-939. 10.1016/j.echo.2014.07.012

Porter TR, Feinstein SB, Ten Cate FJ, and van den Bosch AE. 2020. New Applications in Echocardiography for Ultrasound Contrast Agents in the 21st Century. Ultrasound in Medicine and Biology 46:1071-1081. 10.1016/j.ultrasmedbio.2020.01.004

Raher MJ, Thibault H, Poh KK, Liu R, Halpern EF, Derumeaux G, Ichinose F, Zapol WM, Bloch KD, Picard $\mathrm{MH}$, and Scherrer-Crosbie M. 2007. In Vivo Characterization of Murine Myocardial Perfusion With Myocardial Contrast Echocardiography. Circulation 116:1250-1257. doi:10.1161/CIRCULATIONAHA.107.707737

Scherrer-Crosbie M, Steudel W, Ullrich R, Hunziker PR, Liel-Cohen N, Newell J, Zaroff J, Zapol WM, and Picard MH. 1999. Echocardiographic determination of risk area size in a murine model of myocardial ischemia. American Journal of Physiology-Heart and Circulatory Physiology 277:H986-H992. 10.1152/ajpheart.1999.277.3.H986

Scherrer-Crosbie M, and Thibault HB. 2008. Echocardiography in translational research: of mice and men. Journal of the American Society of Echocardiography : official publication of the American Society of Echocardiography 21:1083-1092. 10.1016/j.echo.2008.07.001

Sirsi SR, Hernandez SL, Zielinski L, Blomback H, Koubaa A, Synder M, Homma S, Kandel JJ, Yamashiro DJ, and Borden MA. 2012. Polyplex-microbubble hybrids for ultrasound-guided plasmid DNA delivery to solid tumors. Journal of Controlled Release 157:224-234. https://doi.org/10.1016/j.jconrel.2011.09.071

Smiseth OA, Torp H, Opdahl A, Haugaa KH, and Urheim S. 2016. Myocardial strain imaging: how useful is it in clinical decision making? European heart journal 37:1196-1207. 10.1093/eurheartj/ehv529

Taqueti VR, and Di Carli MF. 2018. Coronary Microvascular Disease Pathogenic Mechanisms and Therapeutic Options. <span class="subtitle" $><e m>J A C C</$ em $>$ State-of-the-Art Review $</$ span $>$ 72:2625-2641. 10.1016/j.jacc.2018.09.042

Tarnavski O, McMullen JR, Schinke M, Nie Q, Kong S, and Izumo S. 2004. Mouse cardiac surgery: comprehensive techniques for the generation of mouse models of human diseases and their application for genomic studies. Physiological Genomics 16:349-360. 10.1152/physiolgenomics.00041.2003

Theodoropoulos C, and Xu A. 2008. Strain Analysis - An Overview. Visualsonics.

Verkaik M, Poelgeest EMv, Kwekkeboom RFJ, Wee PMt, Brom CEvd, Vervloet MG, and Eringa EC. 2018. Myocardial contrast echocardiography in mice: technical and physiological aspects. American Journal of Physiology-Heart and Circulatory Physiology 314:H381-H391. 10.1152/ajpheart.00242.2017

VisualSonics. VevoCQ ${ }^{\text {TM }}$ Advanced Contrast Quantification Software Analysis Tools for the Vevo ${ }^{\circledR} 2100$ System. VisualSonics. 
Wang F, Nojima M, Inoue Y, Ohtomo K, and Kiryu S. 2015a. Assessment of MRI Contrast Agent Kinetics via Retro-Orbital Injection in Mice: Comparison with Tail Vein Injection. PLOS ONE 10:e0129326. 10.1371/journal.pone.0129326

Wang G, Zhang LE, Manyande A, Cao M, He W, Wu X, Wang J, and Zhang C. 2015b. Effects of anesthesia on conventional and speckle tracking echocardiographic parameters in a mouse model of pressure overload. Experimental and therapeutic medicine 9:1679-1687. 10.3892/etm.2015.2331

Wang J, Bo H, Meng X, Wu Y, Bao Y, and Li Y. 2006. A simple and fast experimental model of myocardial infarction in the mouse. Texas Heart Institute journal 33:290-293.

WHO. 2017. Cardiovascular Diseases (CVDs). Available at https://www.who.int/news-room/factsheets/detail/cardiovascular-diseases-(cvds) (accessed May 7, 2020.

Yardeni T, Eckhaus M, Morris HD, Huizing M, and Hoogstraten-Miller S. 2011. Retro-orbital injections in mice. Lab Animal 40:155-160. 10.1038/laban0511-155 


\section{Figure 1}

Example ultrasound images of the 2D PLAX view of the left ventricle at multiple stages during the process of microbubble injection.

A: 2D B-Mode PLAX of the left ventricle pre-microbubble injection in a sham-operated animal. B: 2D PLAX post-microbubble injection of sham-operated animal as an MIP image, which shows the total accumulation of microbubbles over a period of time to better display the distribution of microbubbles in the myocardium. C: Kinetic graph of the non-linear contrast (NLC) signal of microbubble arrival and plateau in VevoCQ software of a sham-operated animal upon microbubble injection. Locations where WiR and PE were derived from are approximated with red markings. D: 2D B-Mode PLAX of the left ventricle pre-microbubble injection in an MI animal. E: 2D B-Mode PLAX post-microbubble injection of an MI animal as MIP image. F: Kinetic graph of the NLC signal of microbubble arrival and plateau in VevoCQ software of MI animal upon microbubble injection. Locations where WiR and PE were derived from are approximated with red markings. G: Scatter plot of individual data points for the WiR calculated from the slope of the microbubble arrival kinetics in panels $C$ and F. Data represents 1-week and 2-week cohorts of sham-operated animals (blue, $\mathrm{n}=4$ ), $\mathrm{Ml}$ animals (red, $\mathrm{n}=5$ ), and failed Mls (green) with arrows indicating if they were able to be differentiated from the Ml cohort. The failed Mls were excluded from mean data calculations. * $401.86 \pm 140.44, * * 70.32 \pm 95.92$, *** $111.30 \pm 65.12, * * * * 39.27 \pm 26.07$. H: Scatter plot of individual data points for the PE calculated from the plateau of the microbubble arrival kinetics in panels $C$ and $F$. Data represents sham-operated animals (blue, $n=4$ ), MI animals (red, $n=5$ ), and failed Mls (green) with arrows indicating if they were able to be differentiated from the MI cohort. The failed MIs were excluded from mean data calculations. * 442.29 $\pm 163.00, * * 75.08 \pm 27.23, * * * 270.39 \pm 75.01, * * * * 65.68 \pm 23.61$.

MCE: myocardial contrast echocardiography; MI: myocardial infarction; PLAX: parasternal long axis; MIP: maximum intensity projection; NLC: non-linear contrast; PE: peak enhancement; WiR: wash-in-rate. 
A

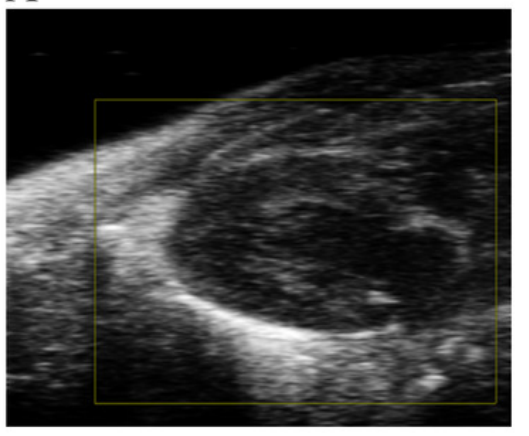

C

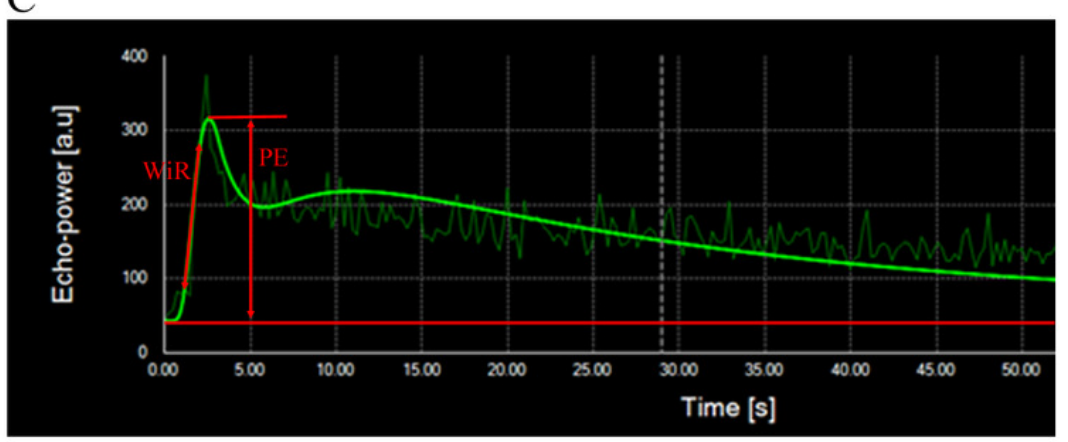

D

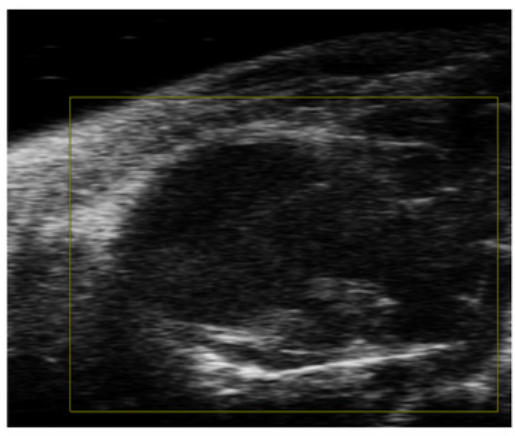

E

F
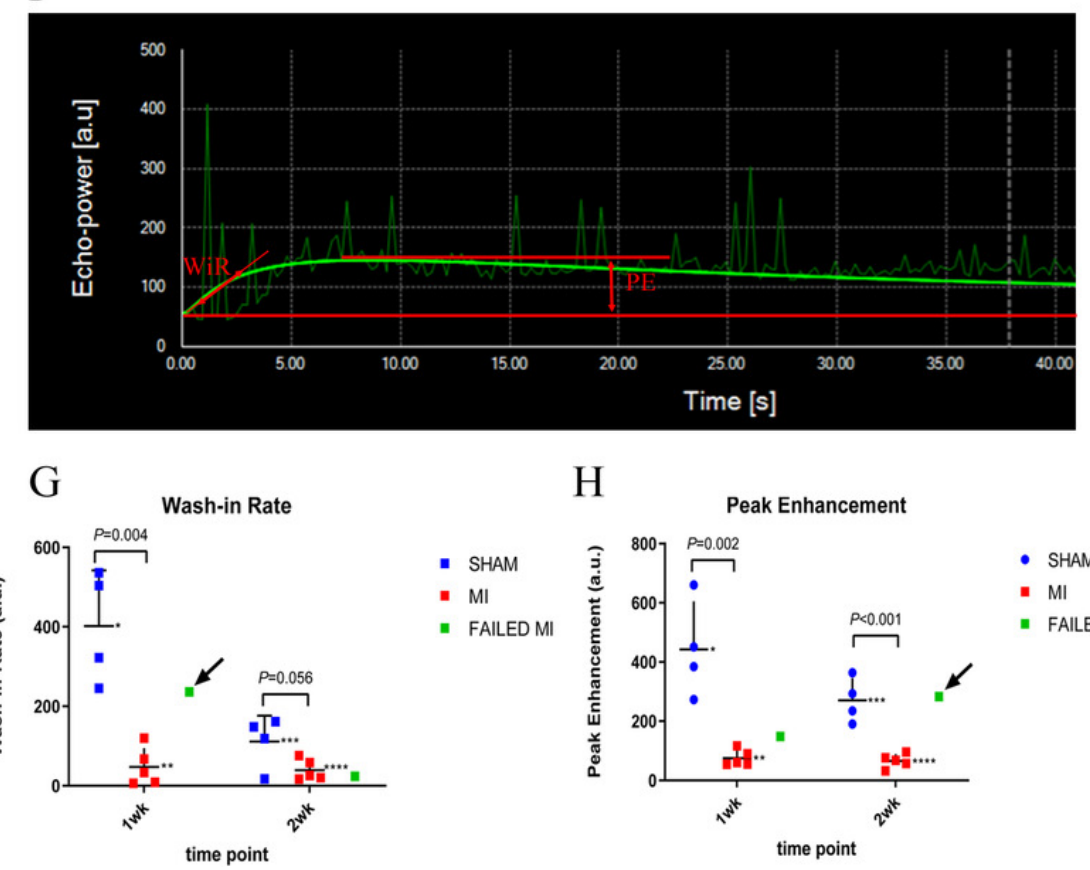

H
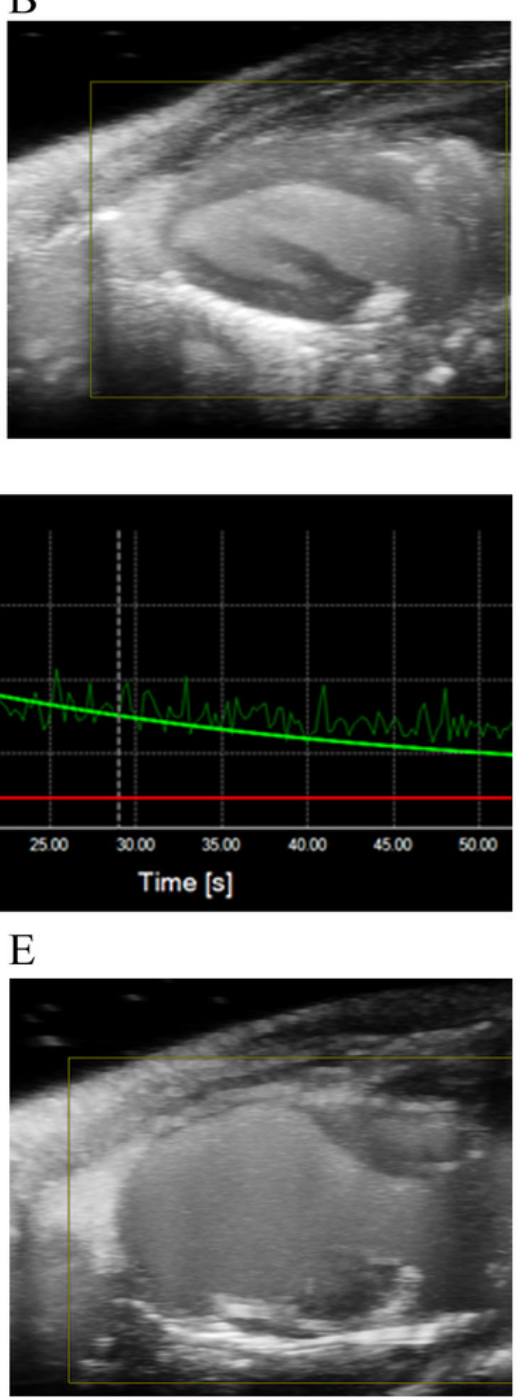

B

\section{.}




\section{Figure 2}

Example image of a 3D myocardial reconstruction and associated PA data.

A: $3 D$ reconstruction of a sham-operated animal indicating the method used to define the myocardial region of interest (ROI) for evaluation of the MCE PA parameter. B: Scatter plot of individual animal PA data. Data represents 1-week and 2-week cohorts of sham-operated animals (blue, n=4), MI animals (red, n=5), and failed MIs (green) with arrows indicating if they were able to be differentiated from the MI cohort. The failed Mls were excluded from mean data calculations. * 73.27 $\pm 10.02, * * 46.32 \pm 11.70, * * * 81.89 \pm 9.49, * * * *$ $42.17 \pm 6.34$.

ROI: region of interest; PA: percent agent; MI: myocardial infarction.

A

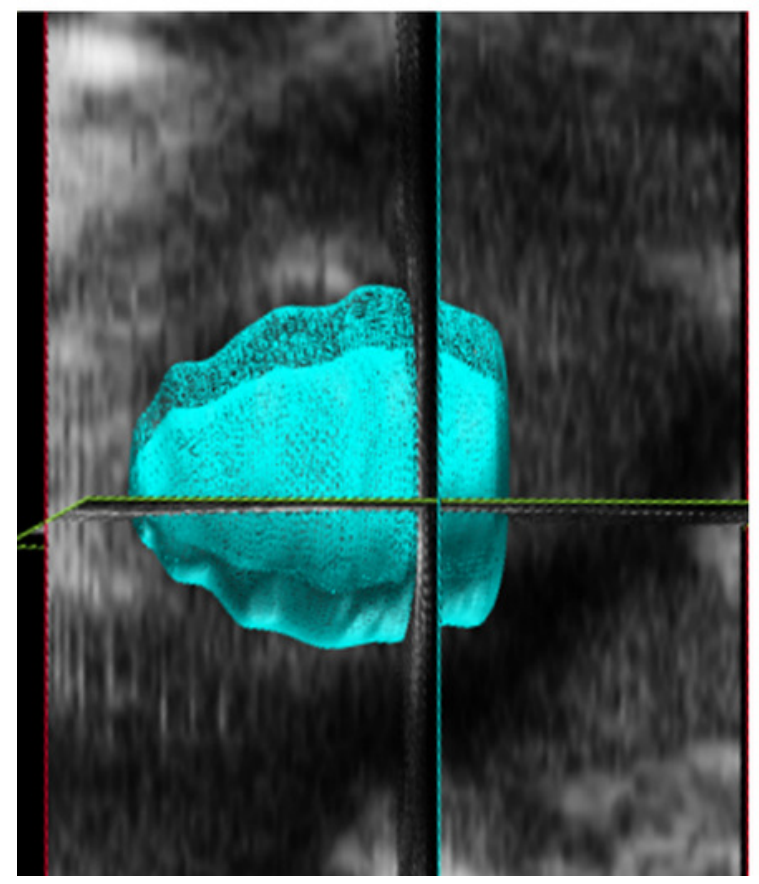

B

\section{Percent Agent}

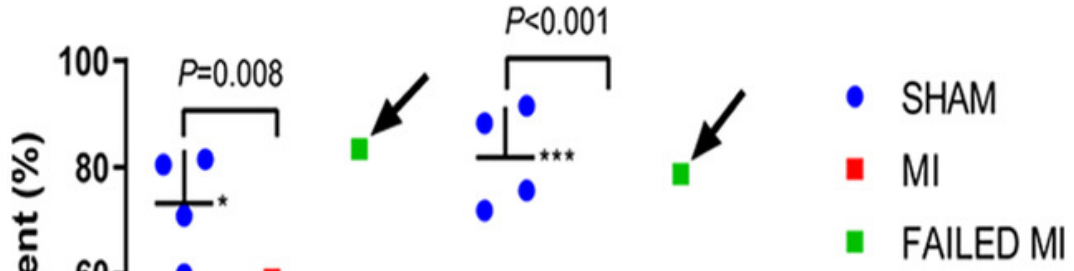




\section{Figure 3}

\section{TTC stained hearts collected at endpoint.}

A: Non-infarcted sham-operated animal showing normal perfusion throughout the myocardium as indicated by the bright red color. B: MI heart showing non-perfused areas as indicated by the white color. C\&D: TTC staining of myocardium which failed to have a significant MI from 1-week and 2-week cohorts, respectively. E: Individual animal data representing the TTC\% infarction. The MI animals (red), sham-operated animals (blue), and failed MI animals (green) are depicted in 1-week and 2-week cohorts separately. The arrows indicate the failed $\mathrm{MI}$ animals that were able to be differentiated from the MI cohort. $\mathrm{n}=4$ for both shamoperated and $\mathrm{MI}$ animals in the 1-week cohort due to the destruction one MI heart during histology preparation. $n=4$ for the sham-operated and $n=5$ for the MI animals in the 2-week cohort. The failed Mls were excluded from mean data calculations. * 1.53 $\pm 1.80, * * 31.64 \pm 4.04, * * * 2.30 \pm 4.24, * * * * 22.04 \pm 5.63$.

TTC: triphenyltetrazolium chloride staining; MI: myocardial infarction. 
A

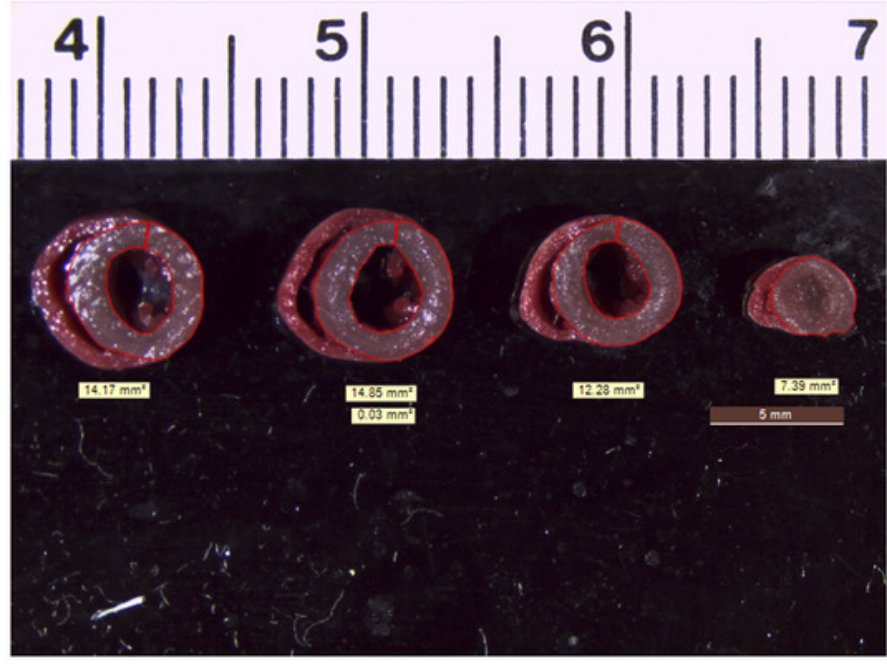

C
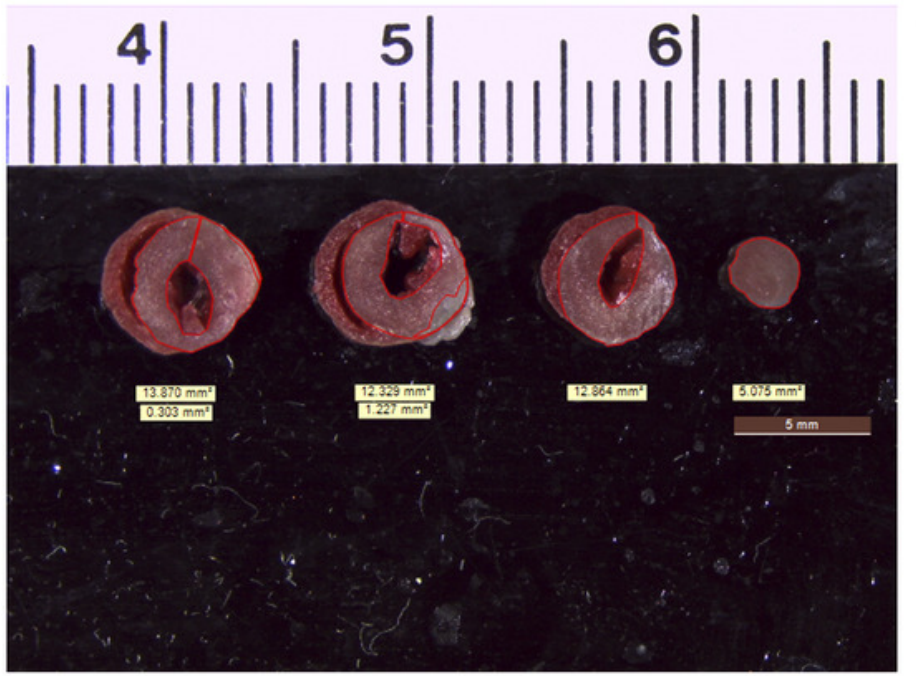

B

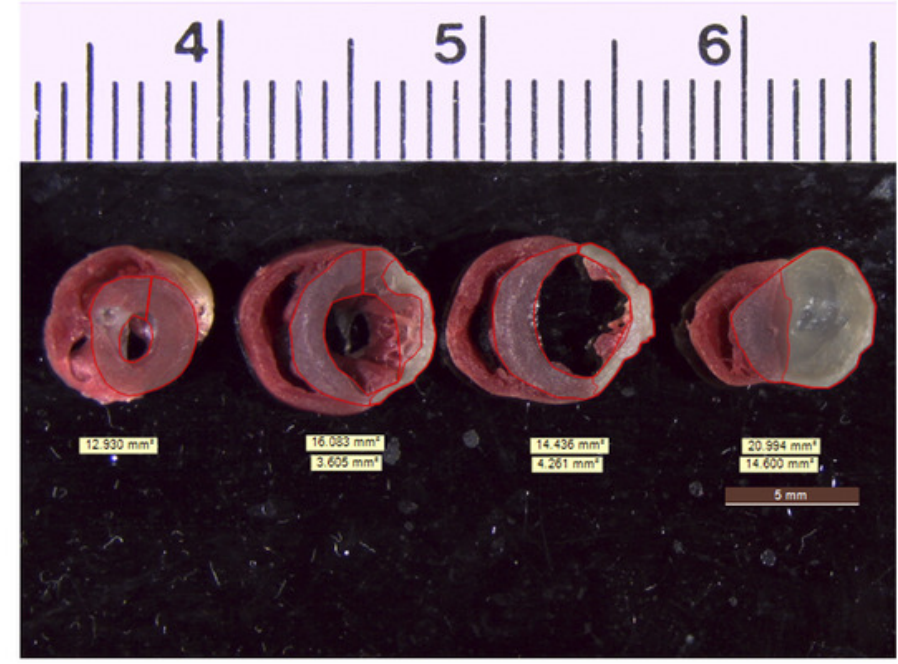

D

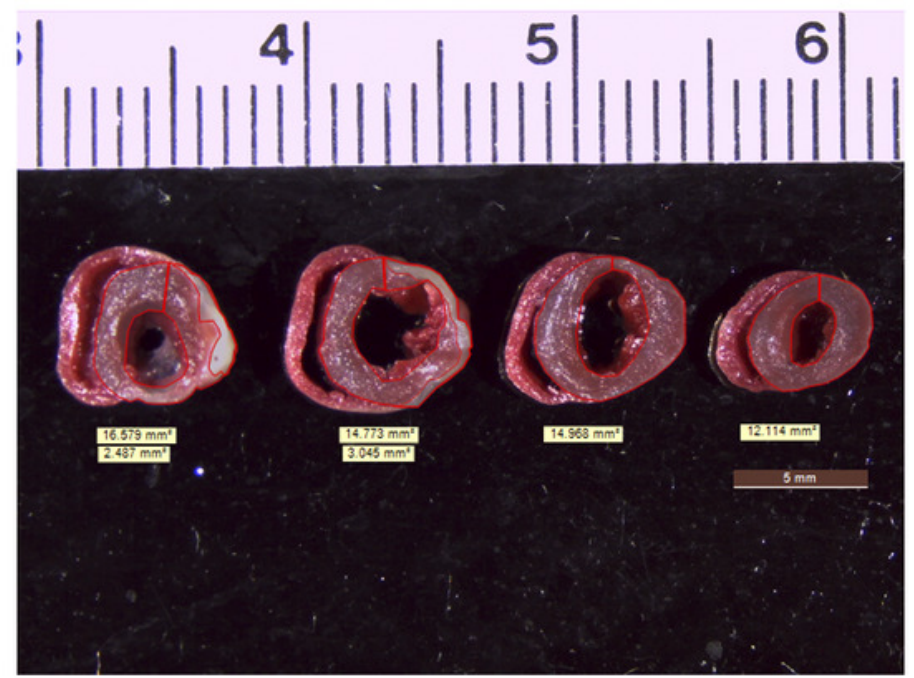

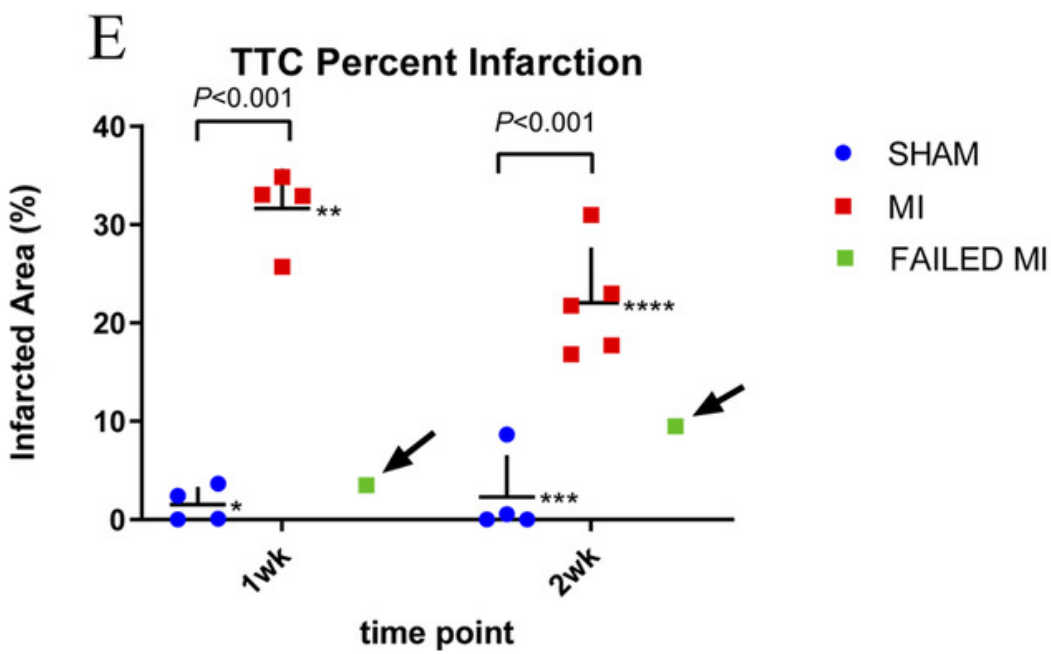




\section{Figure 4}

Scatter plots of individual animal data from conventional echocardiogram techniques of MI (red, $n=5)$, sham-operated (blue, $n=4)$, and failed MI animals (green) in 1-week and 2-week cohorts.

The arrows indicate when a failed MI was able to be differentiated from the MI cohort. Failed Mls were not included in mean data calculations. A: Represents the EF calculated from standard M-Mode. * 60.86 \pm 5.86 , ** 26.05 \pm 12.89 , *** $61.87 \pm 9.06, * * * * 23.20 \pm 12.66$. B: Represents the GLS as calculated from 2D strain analysis. * $-15.57 \pm 4.86, * *-4.90 \pm 1.48, * * *-19.33 \pm 2.27, * * * *-3.08 \pm 1.73$. C: GCS as calculated from $2 \mathrm{D}$ strain analysis. $*-22.35 \pm 3.71, * *-7.83 \pm 3.92, * * *-23.33 \pm 3.85, * * * *-6.83 \pm 5.38$.

MI: myocardial infarction; M-Mode: motion-mode; EF: ejection fraction; GLS: global longitudinal strain; GCS: global circumferential strain. 


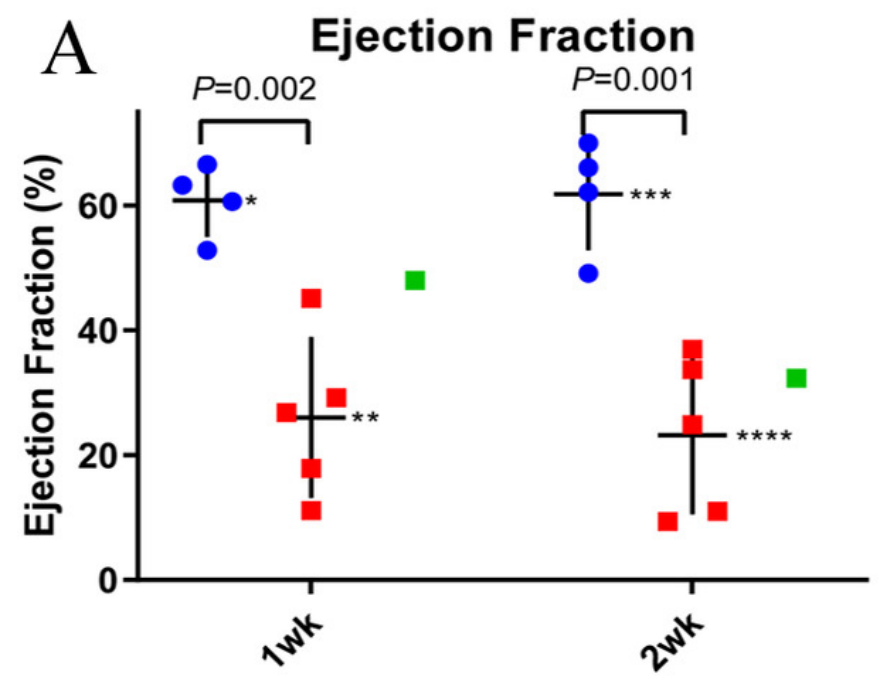

time point

B Global Longitudinal Strain

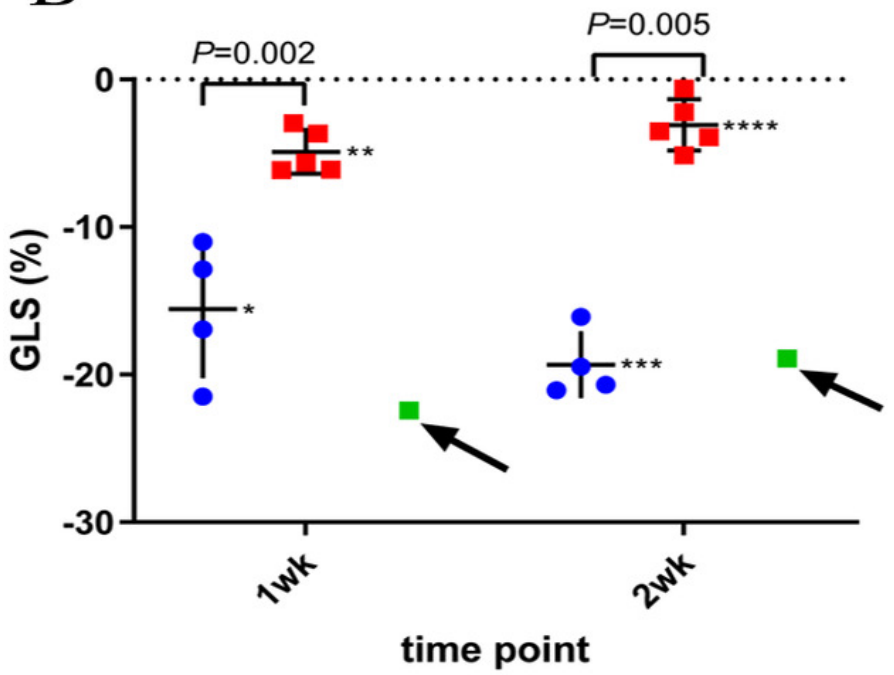

C Global Circumferential Strain

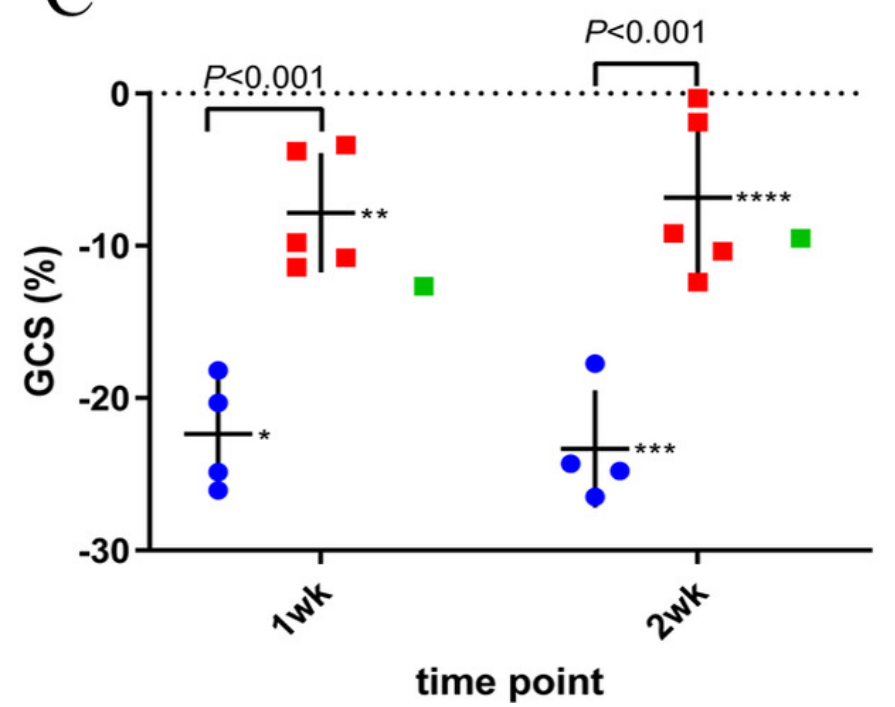

Peer] reviewing PDF | (2020:12:56774:1:1:NEW 10 Apr 2021)
- SHAM

- MI

- FAILED Ml
- SHAM

- MI

- FAILED MI
- SHAM

- $\mathrm{MI}$

- FAILED MI 
Figure 5

Pearson Correlations depicting comparison of TTC staining versus each proposed MCE parameter at the 1-week and 2-week cohort with superimposed $r$ and $P$ values.

$n=4$ for both sham-operated groups, $n=5$ for the 1 week MI group and $n=6$ for the 2 week MI group. A: PE 1-week cohort. B: PE 2-week cohort. C: WiR 1-week cohort. D: WiR 2-week cohort. E: PA 1-week cohort. F: PA 2-week cohort.

MCE: myocardial contrast echocardiography; TTC: triphenyltetrazolium chloride staining; MI: myocardial infarction; PE: peak enhancement; WiR: wash-in-rate; PA: percent agent.

A

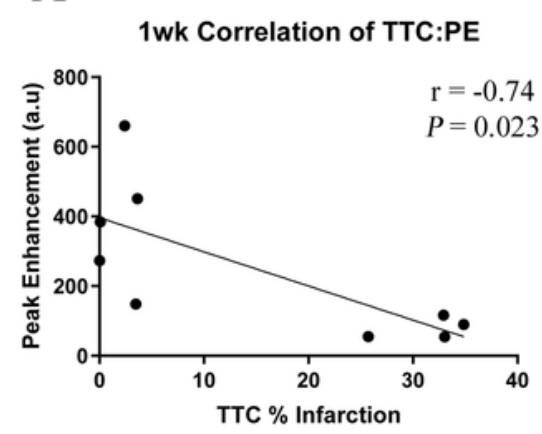

$\mathrm{D}$

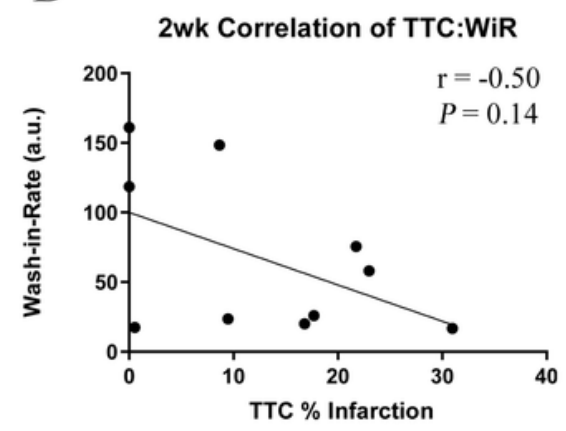

B

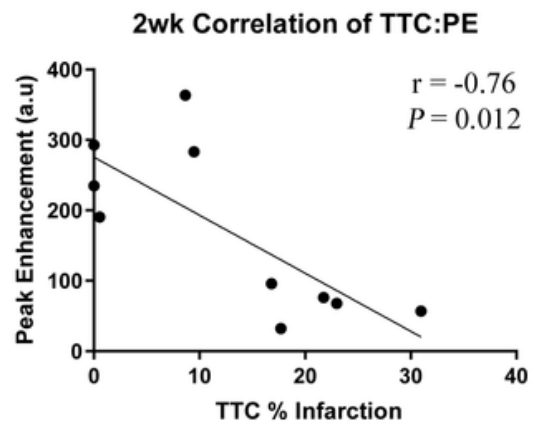

E

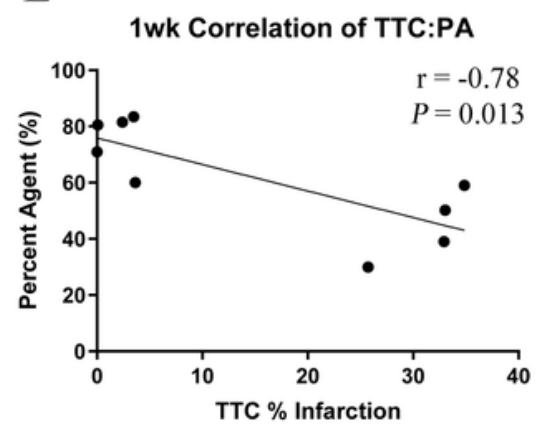

C

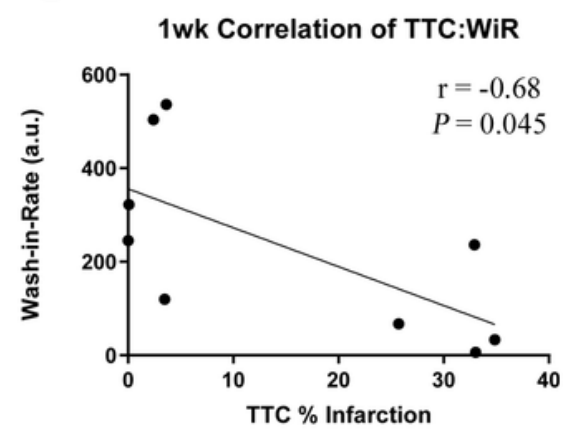

F

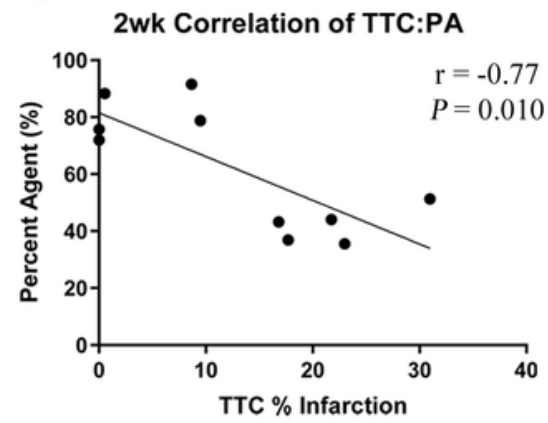




\section{Table $\mathbf{1}$ (on next page)}

Comparison of potential myocardial lodging of neutral size-isolated microbubbles (nSIMB) and cationic size-isolated microbubbles (cSIMB) using the MCE parameters PE and WiR.

Only sham-operated animals were included. 1- and 2-week cohorts were combined for $n=8$ for each cohort of microbubble type.

PE Arrival: peak enhancement plateau shortly after microbubble arrival to the myocardium; PE 8-min: peak enhancement plateau 8-min after microbubble arrival; WiR: wash-in-rate; MCE: myocardial contrast echocardiography; a.u.: arbitrary unit. 
1

\begin{tabular}{|l|l|l|l|}
\hline & nSIMB & cSIMB & $P$ \\
\hline $\begin{array}{l}\text { PE Arrival } \\
\text { [a.u.] }\end{array}$ & $356.34 \pm 149.14$ & $354.58 \pm 185.17$ & 0.98 \\
\hline PE 8-min [a.u.] & $49.35 \pm 47.52$ & $24.64 \pm 19.28$ & 0.19 \\
\hline WiR [a.u.] & $256.58 \pm 185.45$ & $378.08 \pm 334.31$ & 0.38 \\
\hline
\end{tabular}




\section{Table 2(on next page)}

Pearson Correlations of conventional echocardiogram measurements of 2D EF and GLS versus the proposed MCE parameters of PE, WiR, and PA in the specified cohorts.

$\mathrm{n}=4$ for all sham-operated cohorts and $\mathrm{n}=6$ for all MI cohorts.

MCE: myocardial contrast echocardiography; GLS: global longitudinal strain; 2D EF: two-dimensional ejection fraction percent; PE: peak enhancement; WiR: wash-in-rate; PA: percent agent. 


\begin{tabular}{|c|c|c|c|c|c|c|c|c|c|c|c|c|}
\hline & \multicolumn{3}{|c|}{1 week $2 \mathrm{D} \mathrm{EF}$ vs } & \multicolumn{3}{|c|}{2 week $2 \mathrm{D}$ EF vs } & \multicolumn{3}{|c|}{1 week GLS vs } & \multicolumn{3}{|c|}{2 week GLS vs } \\
\hline & PA & $\mathrm{PE}$ & WiR & PA & $\mathrm{PE}$ & WiR & PA & $\mathrm{PE}$ & WiR & PA & $\mathrm{PE}$ & WiR \\
\hline $\mathrm{r}$ & 0.77 & 0.76 & 0.67 & 0.86 & 0.85 & 0.57 & -0.80 & -0.65 & $\begin{array}{l}- \\
0.53\end{array}$ & -0.88 & -0.88 & $\begin{array}{l}- \\
0.52\end{array}$ \\
\hline $\mathrm{CI}$ & $\begin{array}{l}0.27 \\
\text { to } \\
0.94\end{array}$ & $\begin{array}{l}0.25 \\
\text { to } \\
0.94\end{array}$ & $\begin{array}{l}0.06 \\
\text { to } \\
0.91\end{array}$ & $\begin{array}{l}0.51 \\
\text { to } \\
0.97\end{array}$ & $\begin{array}{l}0.48 \\
\text { to } \\
0.96\end{array}$ & $\begin{array}{l}-0.09 \\
\text { to } \\
0.88\end{array}$ & $\begin{array}{l}-0.95 \\
\text { to }- \\
0.35\end{array}$ & $\begin{array}{l}-0.91 \\
\text { to - } \\
0.04\end{array}$ & $\begin{array}{l}- \\
0.87 \\
\text { to } \\
0.15\end{array}$ & $\begin{array}{l}-0.97 \\
\text { to }- \\
0.56\end{array}$ & $\begin{array}{l}-0.97 \\
\text { to - } \\
0.55\end{array}$ & $\begin{array}{l}- \\
0.87 \\
\text { to } \\
0.17\end{array}$ \\
\hline$P$ & 0.009 & 0.011 & 0.036 & 0.002 & 0.002 & 0.086 & 0.005 & 0.042 & 0.11 & $<0.001$ & $<0.001$ & 0.13 \\
\hline
\end{tabular}

